Revista Colombiana de Obstetricia y Ginecología Vol. 56 No.2 • 2005 • (134-140)

INVESTIGACIÓN ORIGINAL

\title{
REGISTRO DE CÁNCER GINECOLÓGICO CLÍNICA SAN PEDRO CLAVER, BOGOTÁ, COLOMBIA 2003
}

\author{
Cancer institutional registry in a gynaecological service \\ at San Pediro Claver Clinic, Bogotá, Colombia 2003
}

Mario A. González, M.D., M.Sc*

Recibido: enero 26/2005 - Revisado: abril 18 de 2005 - Aceptado: mayo 31 de 2005

\section{RESUIMEN}

Objetivo: revisar los casos nuevos de cáncer ginecológico atendidos durante el año 2003 en la Clínica San Pedro Claver, Bogotá, Colombia.

Materiales y métodos: la captación de los casos se hizo a través de la junta de ginecología oncológica a la cual asisten todas las pacientes nuevas de este servicio. Para garantizar la inclusión de la totalidad de ellas, se realizó una búsqueda activa de casos entre las pacientes hospitalizadas, registros de programación de cirugía, reportes de patología y en consulta externa. Los datos fueron registrados en un archivo de Excel y evaluados mediante el programa EPIINFO 2002.

Resultados: se encontraron 158 casos de cáncer ginecológico, siendo el cáncer de cérvix (75 mujeres) el de mayor frecuencia; para efectos del registro, se consideraron dos tipos de tumores de cérvix: los exocervicales (59 casos) y los endocervicales (16 casos). En estas dos localizaciones, la distribución por estadios mostró el mayor número de casos en estado Ib, 49\% en exocérvix y $62 \%$ en los tumores de endocérvix. El tratamiento más frecuentemente indicado en las mujeres con cáncer de cérvix fue la cirugía.

El carcinoma de endometrio, con 46 mujeres, mostró mayoría en estado I (50\%); el tipo histológico más común fue el carcinoma endometrioide (87\%). El tratamiento más usual fue el de cirugía, aplicado en el 93\% de ellos.

* Ginecólogo Oncólogo. Clínica San Pedro Claver. Docente de la Facultad de Medicina de la Fundación Universidad San Martín, Bogotá, Colombia. Correo electrónico: marioar90@hotmail.com
Se encontraron 34 pacientes con carcinoma de ovario, entre los que hubo una proporción igual de casos en estados tempranos I y II que en los tardíos III y IV; el tipo histológico más frecuente fue el seroso $(52,3 \%)$. El tratamiento más usado fue la combinación de cirugía y quimioterapia.

Conclusiones: el registro institucional de cáncer de la Clínica San Pedro Claver es una herramienta útil para conocer la distribución de los diferentes tumores ginecológicos evaluados en esta entidad. Permite establecer sus características personales, clínicas y las modalidades terapéuticas recibidas. El cáncer ginecológico registrado con mayor frecuencia en el año 2003 en esta clínica, fue el de cérvix. Este carcinoma es susceptible de prevención secundaria mediante la citología cérvico-vaginal por lo que se hace imperativa la necesidad de revisar y fortalecer el programa de tamizaje para esta enfermedad.

Palabras clave: cáncer, cáncer cervical, cáncer de endometrio, cáncer de ovario, registros de hospitales.

\section{SUMMARY}

Objective: reviewing new cases of gynaecological cancer attending Clínica San Pedro Claver, Bogotá, Colombia during 2003.

Material and methods: the clinic's oncological gynaecology committee facilitated compiling data as all new patients benefiting from this service are interviewed by it; a search was made during clinical meetings, in consultants' offices, amongst hospitalised patients, of operating room schedules and pathological reports to ensure including all new cases. 
The data so collected was recorded in an excel file; EPIINFO software was then used for analysing it.

Results: there were 158 cases of gynaecological cancer, cervical cancer being encountered most frequently (46\%). This neoplasm was divided into exocervix (59 cases) and endocervix (16 cases) categories; the cancerous stage found most often in both was Ib. Surgery was most often employed as treatment for this type of cancer.

Endometrial cancer was found in 46 women. Stage I (50\%), endometroid cancer (55\%) and surgery (55\%) were the stages, histology and treatment most often found, respectively.

34 patients were found to have ovarian cancer, presenting the same ratio of stages I-II as late stage III-IV; serous histology was most frequently encountered (52.3\%). A combination of surgery and chemotherapy were most often employed for treating this disease.

Conclusions: Clínica San Pedro Claver's institutional records led to establishing the distribution of different gynaecological tumours being evaluated there, establishing related personal and clinical characteristics and the types of therapy being received. Cervical cancer was found to be the gynaecological cancer appearing most frequently during 2003 in Clínica San Pedro Claver. This type of cancer can be detected by pap smear screening (cervical-vaginal cytology), meaning that screening/ prevention programmes for this disease should be given high priority.

Key words: cancer, cancer of the cervix, endometrial cancer, ovary cancer, registries.

\section{INTRODUCCIÓN}

El interés por conocer la distribución y características de la patología oncológica ha fortalecido el desarrollo de los registros de cáncer; de éstos, se aceptan dos modalidades: los poblacionales y los hospitalarios (institucionales). Con el registro poblacional se puede conocer la incidencia y evaluar las acciones de salud pública en cáncer, mientras que los registros institucionales recolectan y eva- lúan la información de una entidad hospitalaria en particular, lo que permite, por medio de la suma de varios registros, conocer las estadísticas de cáncer de una región. ${ }^{1}$

A nivel internacional, se reconoce desde 1960 al registro poblacional de Cali como el único programa de este tipo en el país. En cuanto a los registros institucionales de cáncer, éstos se originaron en Colombia con la creación del Instituto Nacional de Cancerología en 1936, el cual ha continuado su actividad hasta la fecha, aparte de esta entidad el desarrollo de los mencionados registros en otros hospitales ha sido escaso, con la excepción del Hospital Universitario Evaristo García de Cali. ${ }^{2}$

El registro institucional de cáncer de la Clínica San Pedro Claver inició su actividad en el año 2002. Este informe tiene como objetivo describir las pacientes con diagnóstico de cáncer ginecológico que ingresaron para evaluación y manejo durante el año 2003.

\section{MATERIALES Y MÉTODOS}

Tipo de estudio: estudio descriptivo.

Participantes y lugar: información obtenida del registro institucional de cáncer de la Clínica San Pedro Claver, correspondiente a todas las pacientes cuyo cáncer ginecológico fue diagnosticado en esta institución por primera vez en el año 2003.

La Clínica San Pedro Claver es un hospital general de referencia, localizado en Bogotá, Colombia, en la que se atiende pacientes calificados como de alta complejidad, afiliados al Instituto de Seguros Sociales (entidad transformada por el Decreto 1750 del 26 de julio del 2003, que incorporó a la clínica en la Empresa Social del Estado Luis Carlos Galán).

Procedimientos: la captación de los casos para el registro se hizo a través de la junta de ginecología oncológica a la cual asisten todas las pacientes nuevas de este servicio. Para garantizar la inclusión de la totalidad de ellas además, se realizó una búsqueda activa de los casos entre las pacientes hospitalizadas, los registros de programación de cirugía, reportes de patología y en consulta externa, cumpliendo con el 
criterio de exhaustividad (captura de todos los casos nuevos). La fuente de los datos de registro fue la historia clínica y de manera directa la entrevista.

La codificación de morbilidad corresponde a la Clasificación Estadística Internacional de Enfermedades y Problemas Relacionados con la Salud (CIE-10), y para codificar el tipo morfológico o histológico del tumor según el diagnóstico anatomopatológico se siguió la Clasificación Internacional de Enfermedades para Oncología (CIE-0).

Dentro de las diferentes secciones de esta última clasificación merece explicación el significado y uso de "SAI" por su aparición en este trabajo; "SAI" significa "sin otra especificación" y es usado por la CIE-0 para el término topográfico o morfológico que no esté siendo modificado por ningún adjetivo, o cuando va acompañado de un adjetivo no descrito en la clasificación.

Dentro de las modalidades de tratamiento, "Otros" hace referencia a intervenciones terapéuticas como psicología, rehabilitación, cirugía plástica o psiquiatría.

Análisis: los datos fueron registrados en un archivo Excel y evaluados mediante el programa EPIINFO versión 2002. Para presentar los resultados se emplearon medidas estadísticas de posición (porcentaje), tendencia central (promedio aritmético) y de variabilidad (rango y desviación estándar).

\section{RESULTADOS}

El registro de cáncer de la Clínica San Pedro Claver al año 2003 posee datos de 158 pacientes con cáncer ginecológico, cuyo diagnóstico se logró con base en la histología del tumor primario (realizado en la institución, con excepción de dos casos). El tiempo transcurrido entre el diagnóstico y la fecha de inicio de tratamiento fue en promedio de 43,25 días.

La procedencia de las pacientes permite evidenciar que la mayoría de las mujeres evaluadas reside en Bogotá, principalmente en las zonas de Kennedy, Engativá y Suba. El 77\% de ellas tiene teléfono propio en su lugar de habitación.

La tabla resume los principales hallazgos clínicos de este registro.

\section{Cáncer de cérvix}

Fue el tumor ginecológico encontrado con mayor frecuencia en esta institución durante el año 2003, con un total de 75 pacientes $(47,4 \%)$. Para efectos del registro, este tipo de tumor se divide en cáncer de endocérvix, 16 casos $(21,3 \%)$ y cáncer de exocérvix, 59 casos $(78,7 \%)$.

\section{Cáncer de endocérvix}

Edad: en las 16 pacientes con este tumor, la edad promedio fue 43,6 años, rango 36 (29-65) años y desviación estándar de 10,1.

Estado clínico: el estado clínico encontrado más frecuentemente fue el Ib en 10 mujeres (62,5\%); se encontraron 2 pacientes con tumores en estado IIb (12,5\%), 2 en IVb (12,5\%), una con IIIb (6,3\%), y una en la que no se realizó diagnóstico del estadio $(6,3 \%)$.

Tipo histológico: en los casos de cáncer endocervical se presentaron 5 con adenocarcinoma mucinoso (31,2\%), siete con adenocarcinoma no especificado $(43,7 \%)$, tres con carcinoma endometrioide $(18,7 \%)$ y uno con carcinoma papilar $(6,3 \%)$. En cuanto al grado de diferenciación histológica se registraron 6 tumores $(37,5 \%)$ grado 1 (bien diferenciados), 6 (37,5\%) grado 2 (moderadamente diferenciados), y 2 (12,5\%) grado 3 (mal diferenciados). Dos tumores no fueron clasificados, $12,5 \%$.

Tratamiento: se realizó cirugía en 15 (93,8\%) de los 16 casos, y radioterapia posterior a tratamiento en $6(37,5 \%)$. En un caso con estado $\mathrm{Ib}$ además de cirugía se aplicó quimioterapia. De las pacientes con carcinoma estado IVb, a una se le adicionó hormonoterapia y a la otra se le manejó con tratamiento paliativo. La mayoría de las pacientes recibieron una o más modalidades terapéuticas.

\section{Cáncer de exocérvix}

Edad: en las 59 pacientes la edad promedio fue 54 años, rango 61 (32-93) años y desviación estándar de 12,7 . 


\begin{tabular}{|c|c|c|c|c|c|c|}
\hline & & $\begin{array}{l}\text { Endocérvix } \\
\mathrm{n}=16\end{array}$ & $\begin{array}{l}\text { Exocérvix } \\
\mathrm{n}=59\end{array}$ & $\begin{array}{c}\text { Endometrio } \\
\mathrm{n}=46\end{array}$ & $\begin{array}{l}\text { Ovario } \\
\mathrm{n}=34\end{array}$ & \\
\hline \multirow{11}{*}{ Estadio } & I a & & 6 & 6 & 3 & \\
\hline & $\mathrm{I} \mathrm{b}$ & 10 & 29 & 14 & 1 & \\
\hline & I c & & & 3 & 5 & \\
\hline & II a & & & 1 & 1 & \\
\hline & II b & 2 & 8 & 8 & 1 & \\
\hline & II C & & & & 4 & \\
\hline & III a & & 1 & 3 & 2 & \\
\hline & III b & 1 & 9 & 3 & 4 & \\
\hline & III $\mathrm{C}$ & & & & 6 & \\
\hline & IV & 2 & 1 & 1 & 3 & \\
\hline & S.D. & 1 & 5 & 7 & 4 & \\
\hline \multirow{4}{*}{$\begin{array}{c}\text { Tipo } \\
\text { histológico }\end{array}$} & & S A I & Cel. escam. no quer. 24 & A. endometrioide 42 & A. seroso & 11 \\
\hline & & A. mucinoso 5 & Cel. escam. quer. 22 & A. S A I & A. mucinoso & 9 \\
\hline & & $\begin{array}{lr}\text { Carcinoma en- } \\
\text { dometrioide }\end{array}$ & Cel. escam. S A I 10 & & C. SA I & 7 \\
\hline & & Otros & Otros & Otros & Otros & 7 \\
\hline \multirow{4}{*}{$\begin{array}{c}\text { Grado de } \\
\text { diferenciación }\end{array}$} & 1 & 6 & 25 & 24 & 13 & \\
\hline & 2 & 6 & 25 & 14 & 8 & \\
\hline & 3 y 4 & 2 & 4 & 5 & 7 & \\
\hline & S.D. & 2 & 5 & 3 & 6 & \\
\hline \multirow{4}{*}{ Tratamiento } & Cirugía & 15 & 35 & 43 & 33 & \\
\hline & Radiotx & 6 & 34 & 16 & 1 & \\
\hline & Quimiotx & 1 & 9 & 4 & 22 & \\
\hline & Otros & 16 & 53 & 42 & 33 & \\
\hline
\end{tabular}

S.D: Sin dato Radiotx: Radioterapia Quimiotx: Quimioterapia

Estado clínico: el más frecuentemente encontrado fue el Ib con 29 pacientes (49,2\%), seguido del IIIb con $9(15,37 \%)$, estado IIB con $8(13,6 \%)$, estado Ia con $6(10,2 \%)$, IIIa una $(1,7 \%)$ e IVa una $(1,7 \%)$. En cinco casos $(8,5 \%)$ no se estableció clasificación. Tipo histológico: la mayoría de los reportes histológicos, 24 (40,6\%) casos, correspondieron a carcinoma de células grandes escamosas no queratinizante. En 22 casos se reportó un tumor de tipo queratinizante (37,2\%); en los 10 casos (16,9\%) restantes, se reportó carcinoma de células escamosas no especificado.
Con relación al grado de diferenciación histológico se registraron 25 tumores grado $1(42,4 \%)$, 25 grado 2 (42,4\%), hubo 3 casos reportados como grado $3(5,1 \%)$ y uno grado 4 (indiferenciado o anaplásico), 1,7\%. Cinco tumores no fueron clasificados $(8,5 \%)$.

Tratamiento: los procedimientos más usados fueron cirugía y radioterapia, con 35 y 34 pacientes respectivamente, coincidiendo los dos tratamientos en 12 mujeres. La quimioterapia fue aplicada en 9 pacientes. 


\section{Cáncer de endometrio}

Edad: la edad promedio de las 46 pacientes con esta patología fue de 61,24 años, rango 53 (34-87) años y desviación estándar de 10,10 años.

Estado clínico: el estado más frecuente fue el estado Ib con 14 mujeres. Al agrupar a todas las pacientes del estado I (Ia, Ib, Ic) suman un número de 23 pacientes (50\%), el II con 9 (19,5\%) pacientes, estado III con 6 $(12,5 \%)$ y el estado IVb con 1 (2\%), en siete pacientes no se estableció el estado $(15,2 \%)$.

Tipo histológico: el patrón encontrado con mayor frecuencia fue el adenocarcinoma endometrioide en 42 casos (91\%), otros tipos encontrados fueron el adenocarcinoma, SAI en dos casos (4,3\%), un adenocarcinoma papilar, SAI, un caso de carcinoma metastásico y un carcinoma de células claras $(2,1 \%$ para cada tipo, respectivamente).

Con relación al grado de diferenciación histológico se registraron 24 tumores grado 1 (52\%), 14 grado 2 (30,4\%), 3 grado 3 (6,5\%) y dos grado 4 (4\%). Tres tumores no fueron clasificados (6,5\%).

Tratamiento: la cirugía fue el procedimiento realizado más frecuentemente (43 casos que corresponden al 93,4\%), y estuvo acompañada la mayoría de las veces de otro procedimiento. En 16 pacientes se realizó cirugía y radioterapia acompañado de otro tratamiento adicional (34\%), los demás tratamientos tuvieron baja frecuencia.

\section{Cáncer de ovario}

Edad: el promedio de edad en las 34 pacientes con este tipo de cáncer fue 55,06, rango de 38 (38-76) años y desviación estándar de 10,8 años.

Estado clínico: quince de los casos estaban distribuidos en los estados I y II (44\%) y quince en los estados III y IV (44\%). En cuatro casos no se pudo establecer clasificación (11\%).

Tipo histológico: el carcinoma seroso se presentó en 11 casos $(52,3 \%)$, carcinoma mucinoso en 9 $(26,4 \%)$ y 7 casos de carcinoma sin otra especificación (20,5\%). Los demás tipos se encontraron en menor cantidad. En cuanto al grado de diferenciación histológico se registraron 13 tumores grado 1
(38\%), 8 grado 2 (23,5\%) y 7 grado 3 (20,5\%). Seis $(17,6 \%)$ tumores no fueron clasificados.

Tratamiento: la cirugía fue el procedimiento realizado más frecuentemente ( 33 de las 34 pacientes); a $21(61,7 \%)$ pacientes se les adicionó quimioterapia y "otro" tratamiento, y en 11 (32,4\%) casos además de la cirugía se adicionó alguna modalidad de los considerados como "Otros" tratamientos.

\section{Otros cánceres}

\section{Cáncer de vulva}

Los tres casos reportados del tipo carcinoma de células escamosas se clasificaron como estado I en una paciente de 54 años tratada con cirugía, acompañada de otro tratamiento, y dos con estado III, de 70 y 76 años, respectivamente tratadas con cirugía y radioterapia.

\section{Cáncer de vagina}

Se presentó un carcinoma no especificado estado I en una mujer de 86 años que no aceptó tratamiento.

\section{DISCUISIÓN}

Este estudio evalúa pacientes afiliadas al sistema de salud en la modalidad del régimen contributivo, con diagnóstico de cáncer ginecológico en el año 2003 y cuyos datos fueron incluidos en el programa del registro institucional de cáncer de la Clínica San Pedro Claver. La mayoría de las pacientes atendidas fueron habitantes de la ciudad de Bogotá.

La codificación de morbilidad se hizo de acuerdo con la Clasificación Estadística Internacional de Enfermedades y Problemas Relacionados con la Salud (CIE-10). ${ }^{3}$ Para codificar el tipo morfológico o histológico del tumor según el diagnóstico anatomo-patológico se siguió la Clasificación Internacional de Enfermedades para Oncología (CIE-0) en su segunda edición. ${ }^{4}$ La Clasificación Internacional de Enfermedades es un sistema de clasificación utilizado en todo el mundo por los estados miembros de la Organización Mundial de la Salud para la codificación y presentación de las 
estadísticas de morbilidad y mortalidad. La CIE-O fue creada específicamente para describir el cáncer y permite la codificación más detallada de todas las neoplasias según su localización topográfica, histología (morfología), comportamiento y grado de diferenciación.

La distribución de los carcinomas ginecológicos en la población estudiada estuvo encabezada por el carcinoma de cuello uterino, que fue también el más frecuente en el Registro del Instituto Nacional de Cancerología de Colombia, con 14,4\% del total de casos de cáncer en el año 2002.5

Con fines del registro se dividió el cáncer de cérvix de acuerdo a su localización topográfica, considerando así los tumores del exocérvix (78\% de casos) y del endocérvix. La mayoría de casos del primero correspondieron al tipo escamocelular en la clasificación histológica, siendo esta proporción semejante a la encontrada en la literatura universal. ${ }^{6}$

El tipo histológico más frecuente de los carcinomas escamocelulares es el tumor de células escamosas grandes no queratinizantes $(40,6 \%)$, coincidiendo con el estudio de González y cols. en el Instituto Nacional de Cancerología de Colombia; sin embargo, según estos autores la proporción fue más acentuada (48\%) con respecto a los otros tipos histológicos. ${ }^{7}$ Este carcinoma es el más susceptible de cambiar su incidencia con base en programas de prevención secundaria mediante la citología cérvico-vaginal. ${ }^{8}$ En la Clínica San Pedro Claver el cáncer de cérvix se diagnostica en estados más tempranos que en el Instituto Nacional de Cancerología de Colombia, donde se detectó la mayor frecuencia en los estadios IIIb con el $37,6 \%,{ }^{5}$ mientras que en la primera institución el estado Ib fue el más encontrado, tanto en exocérvix como en endocérvix (49,1\% y 64,7\% respectivamente). Lo anterior se sabe que incide en el pronóstico esperado de sobrevida a cinco años, cercana al 80\% para el estado I y de 36\% en el estado III. ${ }^{7}$ Esta diferencia podría deberse a una mejor accesibilidad a los servicios por parte de las afiliadas al seguro social. El tratamiento realizado con más frecuencia fue la cirugía, dada la mayoría del estado Ib..$^{6,9-11}$
La distribución por edad del cáncer de endometrio evidencia que esta entidad es predominantemente una enfermedad de la postmenopausia, cuyo diagnóstico histológico más frecuente es el carcinoma endometrioide. La diferenciación del grado histológico es uno de los indicadores más importantes en el pronóstico; el predominio del grado 1 en este estudio favorece una mejor sobrevida y una menor recurrencia.

La cirugía es el procedimiento más utilizado en este carcinoma que requiere valoración quirúrgica para su estadificación y se acompaña de radioterapia cuando se encuentran factores pronósticos adversos. ${ }^{9}$

El tipo histológico usualmente encontrado en el cáncer de ovario es el seroso, tal como se halló en este registro. ${ }^{9}$ La distribución por estadios en el cáncer de ovario permite observar una frecuencia semejante de los estados I y II, en relación con los estados III y IV; dado que en general la detección del cáncer de ovario ocurre en estados avanzados, el hallazgo de una frecuencia alta en estados tempranos constituye una ventaja desde el punto de vista pronóstico. La sobrevida en estados III y IV es del 9 al 28\% mientras que en el estado I es alrededor del $90 \% .{ }^{9}$ El tratamiento más frecuente corresponde a la combinación de cirugía, quimioterapia y otro tratamiento. Esto se ajusta a la recomendación de buscar citorreducción tumoral quirúrgica como enfoque fundamental en esta patología, ${ }^{12}$ con el uso posterior de quimioterapia en todos los estados en los cánceres epiteliales de ovario; excepto en los estados Ia y Ib bien diferenciados, que no requieren de tratamiento adyuvante. ${ }^{9}$

\section{CONCLUISIONES}

El registro institucional de cáncer de la Clínica San Pedro Claver permite conocer la distribución de los diferentes tumores ginecológicos que son evaluados en esta entidad y establecer sus características personales, clínicas y modalidades terapéuticas recibidas. Dentro de los cánceres ginecológicos el de mayor frecuencia en el año 2003 fue el de cérvix, también encontrado en primer lugar en el Insti- 
tuto Nacional de Cancerología de Colombia. Este carcinoma es susceptible de prevención secundaria mediante la citología cérvico-vaginal por lo cual se deben fortalecer los programas de tamizaje para esta enfermedad.

\section{REFERENCIAS}

1. Dos Santos Silva I. Epidemiología del Cáncer: Principios y Métodos. Lyon: Organización Mundial de la Salud. Agencia Internacional sobre el Cáncer; 1999.

2. Secretaría Distrital de Salud de Bogotá, área de viligancia en Salud. Instituto Nacional de Cancerología. Manual metodológico de normas y procedimientos para la implementación de registros institucionales de cáncer; 2002.

3. Organización Panamericana de la Salud. Clasificación estadística internacional de enfermedades y problemas relacionados con la salud (CIE-10) (décima revisión). Publicación científica No 554. Washington: OPS; 1995.

4. Percy C, Van Holten v, Muir C. Clasificación Internacional de Enfermedades para oncología. Segunda edición. Ginebra: Organización Mundial de la Salud (OMS); 1990.
5. Pardo C, Murillo R, Piñeros M, Castro MA. Casos Nuevos de Cáncer en el Instituto Nacional de Cancerología, Colombia, 2002. Rev Colomb Cancerol 2003;7:4-19.

6. Waggoner SE.Cervical cancer. Lancet 2003;361:221725.

7. González MA, Corso J, Posso H, Sáenz, MC, Martínez G. Carcinoma invasivo de cérvix. Instituto Nacional de Cancerología 1985-1987. Rev Colomb Obstet Ginecol 1994;45:135-40.

8. Stewart BW, Kleihues P (eds). World cancer report. Lyon: IARC press; 2003.

9. González MA. Cáncer Ginecológico. Servigraphic Ltda; 1999.

10. González MA. Cáncer de cérvix. En: Cifuentes R, Lomanto A. Texto de Obstetricia y Ginecología. Bogotá: Editora Guadalupe Ltda; 2004.

11. Instituto Nacional de Cancerología. Guías de Práctica Clínica en Enfermedades Neoplásicas, 2001. P. 41350.

12. Randall TC, Rubin SC. Cytoreductive surgery for ovarian cancer. Surg Clin North Am 2001;81:87183. 